\title{
MJFA MMALSSANOONMA LE

\section{Influence of radiation and viscous dissipation on magnetohydrodynamic Jeffrey fluid over a stretching sheet with convective boundary conditions}

\author{
Syazwani Mohd Zokri a, Nur Syamilah Arifin a, Muhammad Khairul Anuar Mohamed a, \\ Mohd Zuki Salleh ${ }^{\mathrm{a},}$, Abdul Rahman Mohd Kasim ${ }^{\mathrm{a}}$, Wan Nur Syahidah Wan Yusoff ${ }^{\mathrm{a}}$ and \\ Nurul Farahain Mohammad ${ }^{b}$
}

\footnotetext{
a Applied \& Industrial Mathematics Research Group, Faculty of Industrial Science \& Technology, Universiti Malaysia Pahang, 26300 Kuantan, Pahang, Malaysia

${ }^{b}$ Department of Computational and Theoretical Sciences, Kulliyyah of Science, International Islamic University Malaysia, 25200 Kuantan, Pahang,
} Malaysia

* Corresponding author: zuki@ump.edu.my

\section{Article history}

Received 19 February 2017

Accepted 9 August 2017

\begin{abstract}
The present paper focuses on the influence of radiation and viscous dissipation on magnetohydrodynamic (MHD) flow and heat transfer of a Jeffrey fluid over a stretching sheet with convective boundary conditions (CBC). The governing equations are reduced to non-linear ordinary differential equations by using similarity transformation variables and then solved by using RungeKutta-Fehlberg method. The results generated from the numerical computations are presented in the form of tables and graphs for some values of Deborah number, ratio of relaxation to retardation times, Eckert number, radiation parameter and magnetic parameter. It is found that the distribution of fluid velocity is noticeably increased with an increment in Deborah number while the distribution of temperature shows the opposite trend.
\end{abstract}

Keywords: radiation, viscous dissipation, Magnetohydrodynamic (MHD), Jeffrey fluid, stretching sheet

\section{INTRODUCTION}

The convection boundary layer flow problem passing over a stretching sheet is crucial in many practical applications, to be exact when dealing with thermal effects where it happened in numerous industrial outputs processes such as plastic sheet, hot rolling, glass fibre production, paper production and wire drawing. These industrial outputs will undergo the heating and cooling process to produce desirable output. Thus, the understanding of the surface geometry and heat transfer with the purpose of upgrading the quality of final products are very important (Magyari and Keller, 1999).

In the past work, most of the modeling of convection boundary layer flow problem has been discovered by concentrating on the Newtonian fluid. However, such fluids are unable to analyse by a single constitutive relationship between shear stress and strain rate. Hence, by reason of increasing importance in the processing industries including metal and polymer sheet, the non-Newtonian fluid has started to capture the attention of the researchers. On top of that, the non-Newtonian fluid is chosen as it can represent the complex nature fluid existed in this world. There are many non-Newtonian fluid established, however, the non-Newtonian Jeffrey fluid is of interests due to its special characteristics of displaying the features of relaxation to retardation times, where plentiful applications appear in polymer industries (Dalir, 2014; Das et al., 2015; Qasim, 2013). Dilute polymer solution is an example of Jeffrey fluid. It is interesting to mention that the existence of Jeffrey fluid model is basically to overcome the weaknesses arises in Maxwell fluid. In comparison to Jeffrey fluid, Maxwell fluid model can only describe the behavior of relaxation, but not retardation (Farooq et al., 2015).
The primary study on the concept of boundary layer behaviour over solid surface has been documented by Sakiadis (1961). By focusing the similar area of study, Erickson et al. (1966) extended the study with the effect of suction or injection. The flow past a stretching sheet was then performed by Crane (1970) while Gupta and Gupta (1977) considered the same surface geometry with the effect of suction or blowing. Salleh et al. (2010) studied the boundary layer flow and heat transfer over a stretching sheet with Newtonian heating. Next, Hayat et al. (2014) focused on the unsteady flow of Jeffrey fluid past a stretching sheet. Since then, the study of boundary layer flow over a stretching sheet with several effects started to continuously grow. For instance, Hayat et al. (2015) and Babu and Narayana (2016) considered the magnetohydrodynamic (MHD) Jeffrey fluid while thermal radiation effect over stretching sheet has been tackled by Narayana and Babu (2016) and Das et al. (2015). The study of MHD flow is important as it has many applications in industry, engineering and metallurgical processes. The use of electrically conductive polymeric liquid in the process of metallurgy and polymer technology may improve the quality of final product. This is because, cooling rate can be controlled by the proper application of applied magnetic field. Furthermore, the thermal radiation effect has significant role in controlling the heat transfer process specifically at high operating temperature such as gas turbines, nuclear power plant and thermal energy store. On the other hand, the viscous dissipation effect which is generated by the frictional force and is considered as a source of heat has been explored comprehensively by Mohamed et al. (2016); Mustafa et al. (2012) and Zokri et al. (2016).

Motivated to the above investigations, the present study aims to explore the influence of convective boundary conditions on the effect of radiation and viscous dissipation in MHD Jeffrey fluid over a 
stretching sheet. The convective boundary condition is known as the supply of heat through a bounding surface of finite thickness and finite capacity. Moreover, the interface temperature is not known a priori but depends on the intrinsic properties of the system (Merkin, 1994). Recent study on the convectively heated stretching sheet has been done by Mohamed et al. (2015) and Al-Sharif et al. (2016).

\section{MATHEMATICAL FORMULATION}

The steady two-dimensional flow over a stretching sheet immersed in an incompressible and electrically conducting Jeffrey fluid of ambient temperature $T_{\infty}$ is considered. The rectangular Cartesian coordinates $(x, y)$ are used in which $x$ - and $y$-axes are measured parallel to the plate and normal to it, respectively and the fluid occupies the region $y \geq 0$. A uniform magnetic field of strength $B_{0}$ is applied normal to the stretching sheet and oriented to the positive $y$ direction. The magnetic Reynolds number is assumed to be small, accordingly the induced magnetic field is negligible. The physical model and coordinate system of this problem is shown in Figure 1. The partial differential equations describing the flow can be written as

$$
\frac{\partial u}{\partial x}+\frac{\partial v}{\partial y}=0
$$

$$
\begin{array}{r}
u \frac{\partial u}{\partial x}+v \frac{\partial u}{\partial y}=\frac{v}{1+\lambda}\left[\frac{\partial^{2} u}{\partial y^{2}}+\lambda_{1}\left(\begin{array}{c}
u \frac{\partial^{3} u}{\partial x \partial y^{2}}+v \frac{\partial^{3} u}{\partial y^{3}}- \\
\left.\left.\frac{\partial u}{\partial x} \frac{\partial^{2} u}{\partial y^{2}}+\frac{\partial u}{\partial y} \frac{\partial^{2} u}{\partial x \partial y}\right)\right]-\frac{\sigma B_{0}^{2}}{\rho} u
\end{array}\right]\right. \\
u \frac{\partial T}{\partial x}+v \frac{\partial T}{\partial y}=\alpha \frac{\partial^{2} T}{\partial y^{2}}-\frac{1}{\rho C_{p}} \frac{\partial q_{r}}{\partial y}+\frac{v}{C_{p}(1+\lambda)} \\
{\left[\left(\frac{\partial u}{\partial y}\right)^{2}+\lambda_{1}\left(u \frac{\partial u}{\partial y} \frac{\partial^{2} u}{\partial x \partial y}+v \frac{\partial u}{\partial y} \frac{\partial^{2} u}{\partial y^{2}}\right)\right],}
\end{array}
$$

subject to the boundary conditions

$$
\begin{aligned}
& u=u_{w}(x)=a x, \quad v=0, \quad-k \frac{\partial T}{\partial y}=h_{f}\left(T_{f}-T\right) \text { at } y=0 \\
& u \rightarrow 0, \quad v \rightarrow 0, \quad T \rightarrow T_{\infty} \text { as } y \rightarrow \infty
\end{aligned}
$$

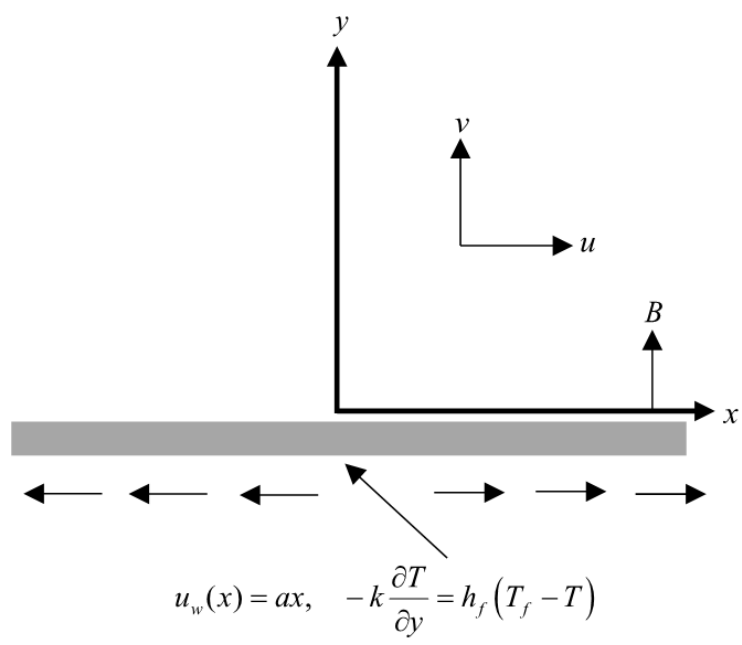

Fig. 1 Physical model of the coordinate system.

where $u_{w}(x)$ is the stretching velocity with $a$ being the positive constant, while $u$ and $v$ are the velocity components along the $x-$ and $y$-directions, respectively. Additionally, $\mu$ is the dynamic viscosity, $v$ is the kinematic viscosity, $\lambda$ is the ratio of relaxation and retardation times, $\lambda_{1}$ is the relaxation time, $g$ is the gravity acceleration, $\alpha$ is the thermal diffusivity, $\beta$ is the thermal expansion,
$T$ is the local temperature, $\rho$ is the fluid density, $\sigma$ is the electric conductivity, $q_{r}$ is the radiative heat flux, $C_{p}$ is the specific heat capacity at a constant pressure, $k$ is the thermal conductivity, $h_{f}$ is the heat transfer coefficient and $T_{f}=T_{\infty}+b x^{2}$ is the hot fluid temperature where $b$ corresponds to dimensionless constant (Afridi et al., 2016).

Imposing the Rosseland approximation for radiation (Bataller, 2008), the radiative heat flux gives

$q_{r}=-\frac{4 \sigma^{*}}{3 k^{*}} \frac{\partial T^{4}}{\partial y}$

where $\sigma^{*}$ and $k^{*}$ are the Stefan-Boltzmann constant and the mean absorption coefficient, respectively. The temperature difference is assumed such that the linear function of temperature is expressed as $T^{4}$. By using Taylor series, the term $T^{4}$ is expanding about $T_{\infty}$ and higher-order terms is neglected. This yields

$T^{4} \cong 4 T_{\infty}^{3} T-3 T_{\infty}^{4}$

Using Eqs. (5) and (6), Eq. (3) reduces to

$$
\begin{aligned}
u \frac{\partial T}{\partial x}+v \frac{\partial T}{\partial y}= & \left(\frac{k}{\rho C_{p}}+\frac{16 \sigma^{*} T_{\infty}^{3}}{3 k^{*} \rho C_{p}}\right) \frac{\partial^{2} T}{\partial y^{2}}+ \\
& \frac{v}{C_{p}(1+\lambda)}\left[\left(\frac{\partial u}{\partial y}\right)^{2}+\lambda_{1}\left(u \frac{\partial u}{\partial y} \frac{\partial^{2} u}{\partial x \partial y}+v \frac{\partial u}{\partial y} \frac{\partial^{2} u}{\partial y^{2}}\right)\right]
\end{aligned}
$$

The influence of radiation in Eq. (7) is expected to enhance the thermal diffusivity. Thus, by expressing the radiation parameter as $N_{R}=\frac{4 \sigma^{*} T_{\infty}^{3}}{k^{*} k}$, Eq. (7) becomes

$$
\begin{aligned}
u \frac{\partial T}{\partial x}+v \frac{\partial T}{\partial y}= & \frac{k}{\rho C_{p}}\left(1+\frac{4 N_{R}}{3}\right) \frac{\partial^{2} T}{\partial y^{2}}+ \\
& \frac{v}{C_{p}(1+\lambda)}\left[\left(\frac{\partial u}{\partial y}\right)^{2}+\lambda_{1}\left(u \frac{\partial u}{\partial y} \frac{\partial^{2} u}{\partial x \partial y}+v \frac{\partial u}{\partial y} \frac{\partial^{2} u}{\partial y^{2}}\right)\right]
\end{aligned}
$$

Introducing the similarity transformation variables:

$$
\eta=\left(\frac{a}{v}\right)^{1 / 2} y, \quad \psi=(a v)^{1 / 2} x f(\eta), \quad \theta(\eta)=\frac{T-T_{\infty}}{T_{f}-T_{\infty}}(\mathrm{CBC})
$$

The Eq. (1) is automatically satisfied if stream function $\psi$ is chosen such that $u=\frac{\partial \psi}{\partial y}$ and $v=-\frac{\partial \psi}{\partial x}$. Then, we have

$$
u=a x f^{\prime}(\eta), \quad v=-(a v)^{1 / 2} f(\eta)
$$

where prime denotes differentiation with respect to $\eta$. By substituting Eqs. (9) and (10) into (2) and (3), we obtain the following nonlinear ordinary differential equations:

$$
\begin{aligned}
& f^{\prime \prime \prime}+(1+\lambda)\left(f f^{\prime \prime}-f^{\prime 2}\right)+\lambda_{2}\left(f^{\prime \prime 2}-f f^{(i v)}\right)-(1+\lambda) M f^{\prime}=0 \\
& (1+\lambda)\left(\left(1+\frac{4 N_{R}}{3}\right) \theta^{\prime \prime}+\operatorname{Pr} f \theta^{\prime}-2 \operatorname{Pr} f^{\prime} \theta\right)+ \\
& E c \operatorname{Pr}\left(f^{\prime \prime 2}+\lambda_{2} f^{\prime \prime}\left(f f^{\prime \prime}-f f^{\prime \prime \prime}\right)\right)=0
\end{aligned}
$$

The boundary conditions Eq. (4) become 
$f(0)=0, \quad f^{\prime}(0)=1, \quad \theta^{\prime}(0)=-B i(1-\theta(0)) \quad$ at $\quad y=0$

$f^{\prime}(\infty) \rightarrow 0, \quad f^{\prime \prime}(\infty) \rightarrow 0, \quad \theta(\infty) \rightarrow 0 \quad$ as $\quad y \rightarrow \infty$

where $B i=\frac{h_{f}}{k}\left(\frac{v}{a}\right)^{1 / 2}$ is the Biot number for convective boundary condition, $\operatorname{Pr}=\frac{\mu}{\alpha \rho}$ is the Prandtl number, $\lambda_{2}=\lambda_{1} a$ is Deborah number, $E c=\frac{u_{w}^{2}}{C_{p}\left(T_{f}-T_{\infty}\right)}=\frac{a^{2}}{b C_{p}}$ is an Eckert number and $M=\frac{\sigma B_{0}^{2}}{\rho a}$ is magnetic parameter. It is worth mentioning that when $B i=0$ and $B i \rightarrow \infty$, the insulated wall and constant wall temperature is presented, respectively. By letting $M=0$, the exact analytical solution of Eq. (11) as given by Hayat et al. (2014) is obtained as follows

$f(\eta)=\frac{1-e^{-m \eta}}{m}$

where $m=\left(\frac{1+\lambda}{1+\lambda_{2}}\right)^{1 / 2}$. Noted that the second derivative of the exact solution for Eq. (14) is $f^{\prime \prime}(\eta)=-m e^{-m \eta}$, therefore, the dimensionless velocity gradient at the sheet surface is

$f^{\prime \prime}(0)=-m$

In practical applications, the physical quantities of interest are the local skin friction coefficient $C_{f}$ and the Nusselt number $N u_{x}$ (Molla et al., 2006):

$C_{f}=\frac{\tau_{w}}{\rho U_{\infty}^{2}}, \quad N u_{x}=\frac{a q_{w}}{k\left(T_{w}-T_{\infty}\right)}$.

The surface shear stress $\tau_{w}$ and the surface heat flux $q_{w}$ are given by (Das et al., 2015)

$\tau_{w}=\frac{\mu}{1+\lambda}\left[\frac{\partial u}{\partial y}+\lambda_{1}\left(u \frac{\partial^{2} u}{\partial x \partial y}+v \frac{\partial^{2} u}{\partial y^{2}}\right)\right]_{y=0}, q_{w}=-k\left(\frac{\partial T}{\partial y}\right)_{\bar{y}=0}$

where $\mu=\rho v$ and $k$ being the dynamic viscosity and the thermal conductivity, respectively. Using Eqs. (16) and (17), the reduced skin friction coefficient $C_{f} \operatorname{Re}_{x}^{1 / 2}$ and reduced Nusselt number $N u_{x} \operatorname{Re}^{-1 / 2}$ are:

$C_{f} \operatorname{Re}_{x}^{1 / 2}=\frac{1+\lambda_{2}}{1+\lambda} f^{\prime \prime}(0)$

and

$N u_{x} \operatorname{Re}_{x}^{-1 / 2}=-\left(1+\frac{4 N_{R}}{3}\right) \theta^{\prime}(0)$

where $\operatorname{Re}=\frac{u_{w} x}{v}$ is the local Reynolds number.

\section{RESULTS AND DISCUSSION}

Eqs. (11) and (12) subject to the boundary conditions (13) were solved numerically by using Runge-Kutta-Fehlberg Method, which is programmed in MAPLE software. The boundary layer thickness in the range of 5 to 30 are used in this study to achieve the far field boundary conditions asymptotically. The fixed parameters used in the simulations are $\operatorname{Pr}=0.71$ (air), $\lambda=\lambda_{2}=0.1, E c=N_{R}=0.2 \quad$ and $\quad B i=M=1.0$, except otherwise stated. Table 1 shows the comparison of the present study with the exact solution of skin friction coefficient $f^{\prime \prime}(0)$ from Eq. (15) and the existing publication documented by Dalir (2014). From this table, it is shown that the results are in great consistency, thus this gives assurance to the authors to proceed with the present codes.

Table 1 Comparison between the present solution and previous published results for various values of $f^{\prime \prime}(0)$ when $\lambda=0.2, \quad M=0$ and $B i \rightarrow \infty$.

\begin{tabular}{cccc}
\hline$\lambda_{2}$ & Exact solution Eq. (15) & Dalir (2014) & Present \\
\hline 0.0 & -1.09544512 & -1.09641580 & -1.09544512 \\
0.2 & -1.00000000 & -1.00124052 & -1.00000000 \\
0.4 & -0.92582010 & -0.92724220 & -0.92582010 \\
0.6 & -0.86602540 & -0.86755715 & -0.86602540 \\
0.8 & -0.81649658 & -0.81808091 & -0.81649658 \\
1.0 & -0.77459667 & -0.77618697 & -0.77459667 \\
1.2 & -0.73854895 & -0.74010502 & -0.73854896 \\
1.4 & -0.70710678 & -0.70859214 & -0.70710680 \\
1.6 & -0.67936622 & -0.68074654 & -0.67936625 \\
1.8 & -0.65465367 & -0.65589608 & -0.65465372 \\
2.0 & -0.63245553 & -0.63352833 & -0.63245560 \\
\hline
\end{tabular}

Fig. 2 demonstrates the variation of wall temperature, $\theta(0)$ with ratio of relaxation to retardation, $\lambda$ when Deborah number, $\lambda_{2}=0.1$ and 1.0. It is found that $\lambda$ is dependent on $\lambda_{2}$, where $\lambda$ must be lesser than some critical value, namely $\lambda_{c}$ in order to attain the physically acceptable solution. Noticeably, $\theta(0)$ increases as $\lambda$ come close to the critical values $\lambda_{c}=2.47060237$ and $\lambda_{c}=7.30094927$ when $\lambda_{2}=0.1$ and $\lambda_{2}=1.0$, respectively. As such, it is concluded that as $\lambda_{2}$ gets larger, the $\lambda_{c}$ will be increased.

Figs. 3 to 8 are plotted to explain the influence of several emerging parameters, i.e. the ratio of relaxation to retardation times $\lambda$, the Deborah number $\lambda_{2}$ and the magnetic parameter $M$ on the velocity and temperature profile, respectively. Meanwhile, Fig. 9 and 10 attempted to describe the effect of the Eckert number $E c$ and the radiation parameter $N_{R}$ on the temperature profile. The velocity profile for these parameters is not plotted as the graph generated is unique, because of decoupled boundary layer Eqs. (11) and (12). Correspondingly, there exist one solution for skin friction coefficient, i.e. $f^{\prime \prime}(0)=-1.41421356$

Fig. 3 presents the effect of $\lambda$ on the $f^{\prime}(\eta)$, where increasing $\lambda$ tends to retard the flow. This will increase the drag force and accordingly the reduction in velocity happens. In Fig. 4, the temperature profile is seen to increase as $\lambda$ increase. Noted that an increase in relaxation time will decrease the retardation time. Since the decrease in retardation time is overriding the increase in relaxation time, thus this results in higher temperature, thereby thickening the thermal boundary layer thickness.

For larger $\lambda_{2}$, the velocity of fluid is noticed to increase as shown in Fig. 5. Oppositely, in Fig. 6, the dimensionless temperature $\theta(\eta)$ is observed to slightly retard as $\lambda_{2}$ increases. This can be directly related to the definition of Deborah number, i.e. $\lambda_{2}=\lambda_{1} a$ where $\lambda_{2}$ is depending on the relaxation time, $\lambda_{1}$. Hence, higher $\lambda_{1}$ causes the increment in $\lambda_{2}$, which in turn increasing the velocity of fluid while decreasing the temperature. Also, for high $\lambda_{2}$, the materials behave rather elastically. 
The effect of magnetic parameter, $M$ on velocity and temperature distributions are performed in Figs. 7 and 8. From Fig. 7, it is observed that as $M$ increases, the velocity profile is decreases. Physically, higher $M$ will strengthen the magnetic field strength. Its presence will produce a bulk namely Lorentz force, that consequently making massive reduction to the velocity of fluid and thus, lessening the momentum boundary layer. On the contrary, the dimensionless temperature in Fig. 8 is enhanced due to an increase in $M$

Fig. 9 shows the temperature profile $\theta(\eta)$ against $\eta$ for several values of $E c$. It is noticed that as $E c$ increases, the temperature profile is also increases owing to the effect of frictional forces that produce heat energy in the fluid.

The influence of radiation parameter on dimensionless temperature is illustrated in Fig. 10, where the temperature is an increasing function of $N_{R}$. An increase in $N_{R}$ delivers extra heat to the fluid that results in the enhancement of temperature and thermal boundary layer thickness.

Figs. 11 to 13 display the influence of the $M, \lambda_{2}, B i$ and $N_{R}$ on the skin friction coefficient $C_{f} \operatorname{Re}_{x}^{1 / 2}$ and local Nusselt number $N u_{x} \mathrm{Re}_{x}^{-1 / 2}$, respectively. In Figs. 11 and 12, the increase of $M$ and $\lambda_{2}$ are found to decrease the $C_{f} \operatorname{Re}_{x}^{1 / 2}$ while increasing the $N u_{x} \mathrm{Re}_{x}^{-1 / 2}$. On the other hand, the $N u_{x} \operatorname{Re}_{x}^{-1 / 2}$ is reduced for larger $B i$ and $N_{R}$ as shown in Fig. 13, representing the depletion of convective heat transfer rate. Moreover, this study reveals that the graph is unique for the $C_{f} \operatorname{Re}_{x}^{1 / 2}$, therefore, the presence of $N_{R}$ and $B i$ are concluded to not possessing any influence to the drag force of fluid.

Several parameters that pronounce significant influences on the numerical values of $C_{f} \mathrm{Re}_{x}^{1 / 2}$ and $N u_{x} \mathrm{Re}_{x}^{-1 / 2}$ are tabulated in Tables 2 and 3. From Table 2, it is observed that as $\lambda_{2}$ and $M$ increase, the $C_{f} \mathrm{Re}_{x}^{1 / 2}$ decreases whereas an increase in $\lambda$ boosts up the $C_{f} \operatorname{Re}_{x}^{1 / 2}$. In Table 3, the heat transfer rate is increase when $\lambda, E c$ and $M$ are increased. Meanwhile, the weak heat transfer is detected for larger values of $B i, \lambda_{2}, \operatorname{Pr}$ and $N_{R}$.

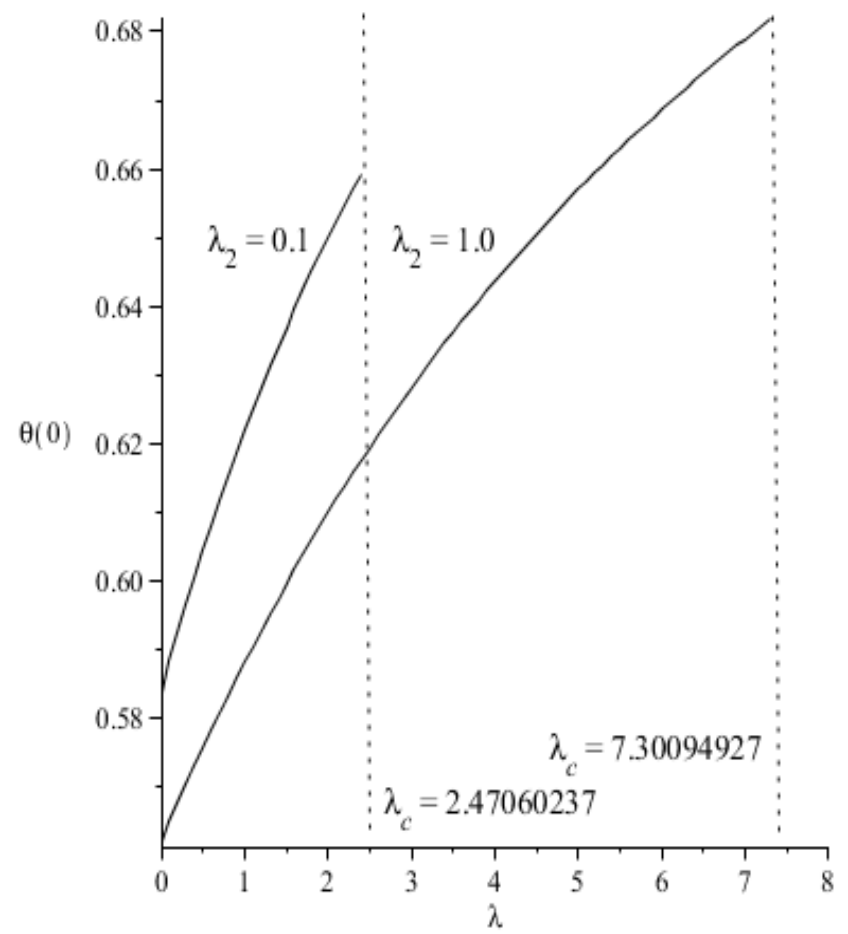

Fig. 2 Variation of the $\theta(0)$ with $\lambda$ when $\lambda_{2}=0.1$ and $\lambda_{2}=1.0$.

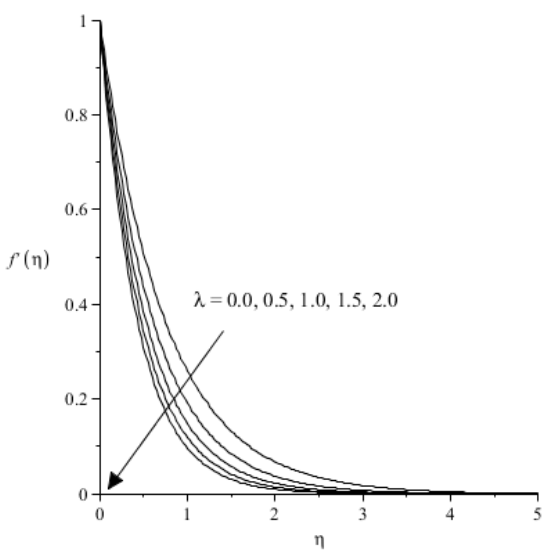

Fig. 3 Velocity profile $f^{\prime}(\eta)$ for various values of $\lambda$.

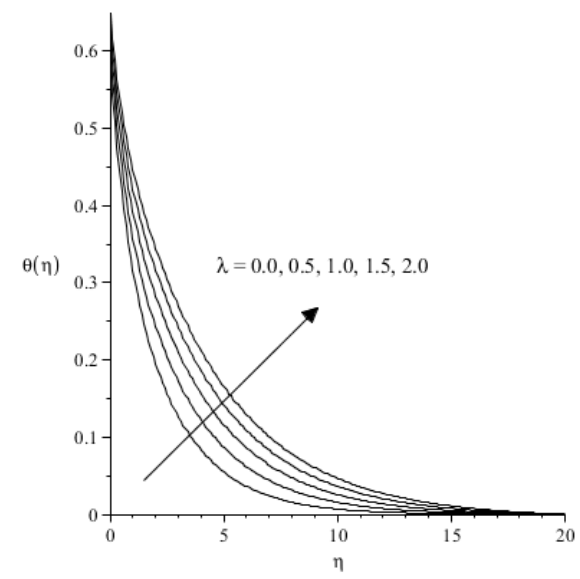

Fig. 4 Temperature profile $\theta(\eta)$ for various values of $\lambda$.

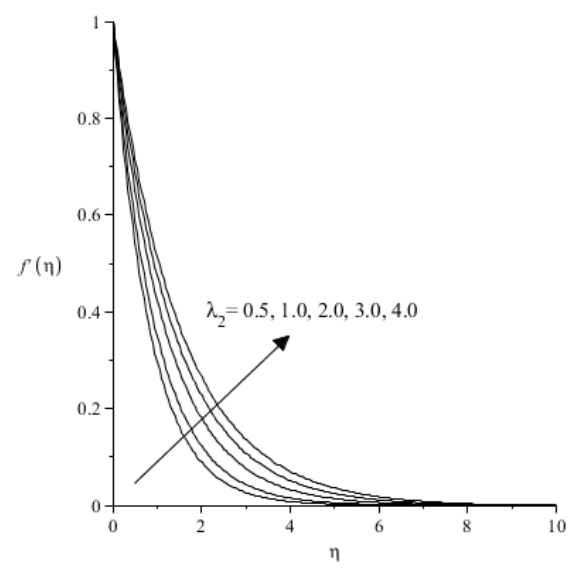

Fig. 5 Velocity profile $f^{\prime}(\eta)$ for various values of $\lambda_{2}$.

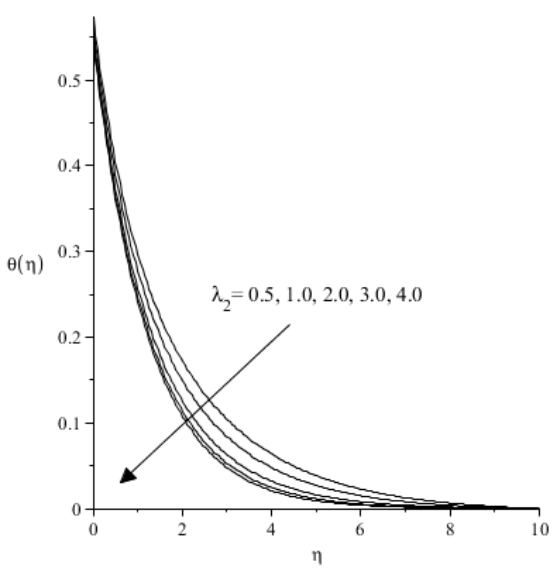

Fig. 6 Temperature profile $\theta(\eta)$ for various values of $\lambda_{2}$. 


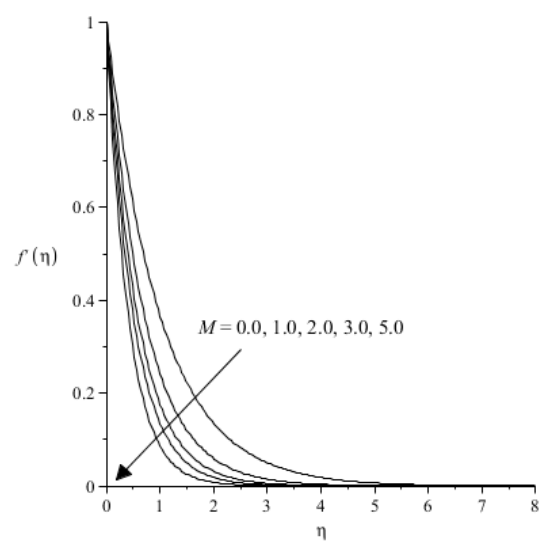

Fig. 7 Velocity profile $f^{\prime}(\eta)$ for various values of $M$.

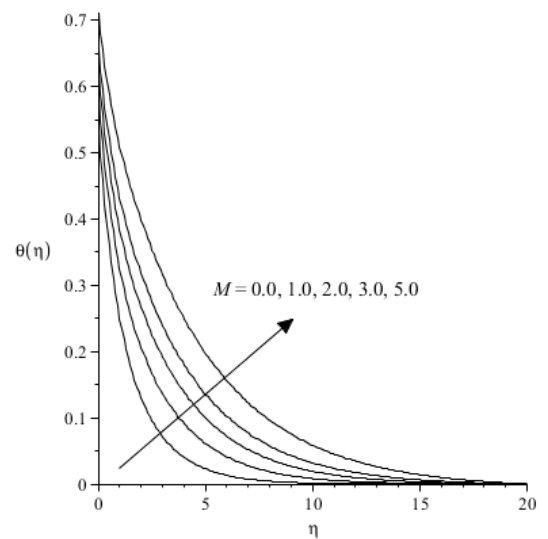

Fig. 8 Temperature profile $\theta(\eta)$ for various values of $M$.

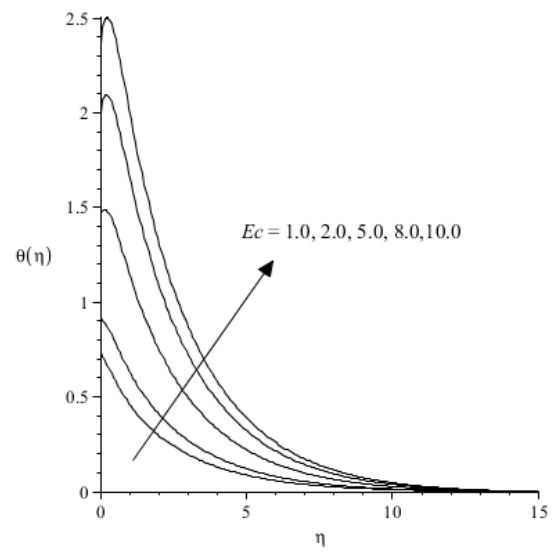

Fig. 9 Temperature profile $\theta(\eta)$ for various values of $E c$.

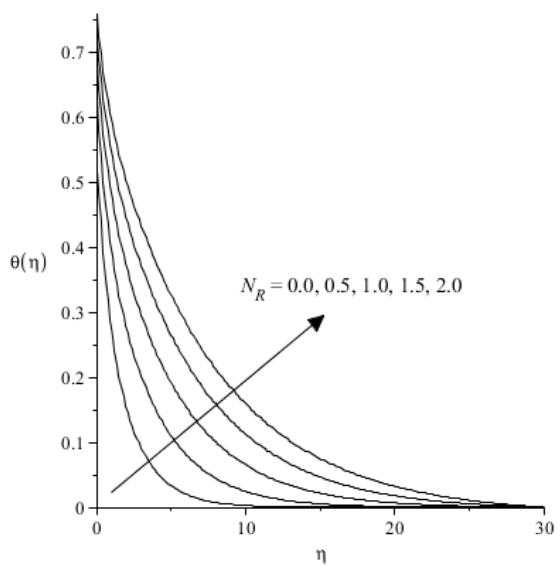

Fig. 10 Temperature profile $\theta(\eta)$ for various values of $N_{R}$.

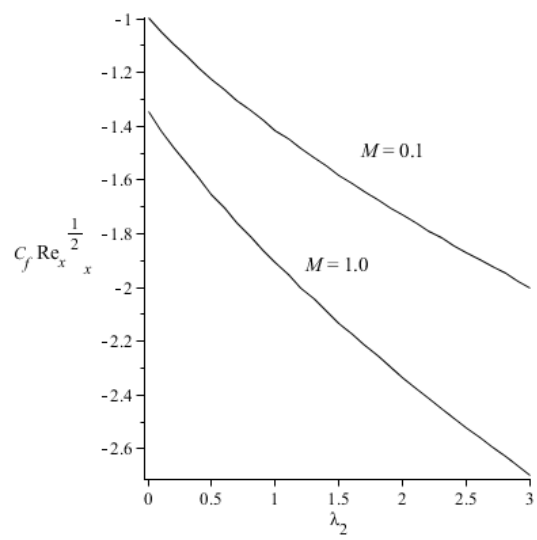

Fig. 11 Variation of the $C_{f} \operatorname{Re}_{x}^{1 / 2}$ with $\lambda_{2}$ for various values of $M$.

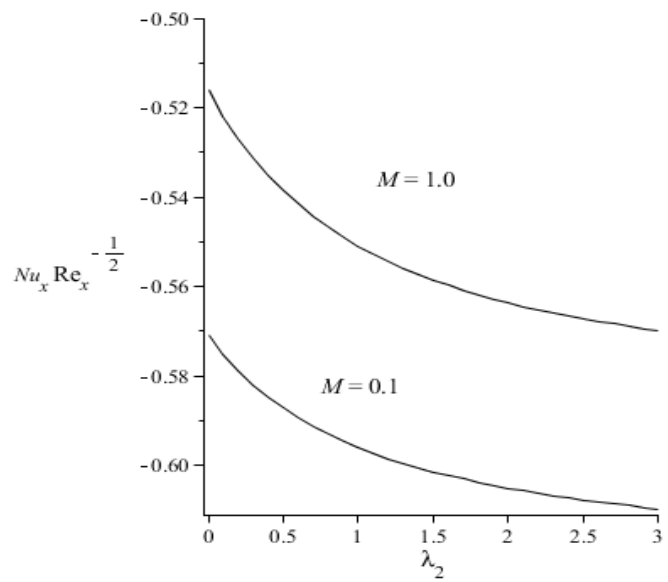

Fig. 12 Variation of the $N u_{x} \mathrm{Re}_{x}^{-1 / 2}$ with $\lambda_{2}$ for various values of $M$.

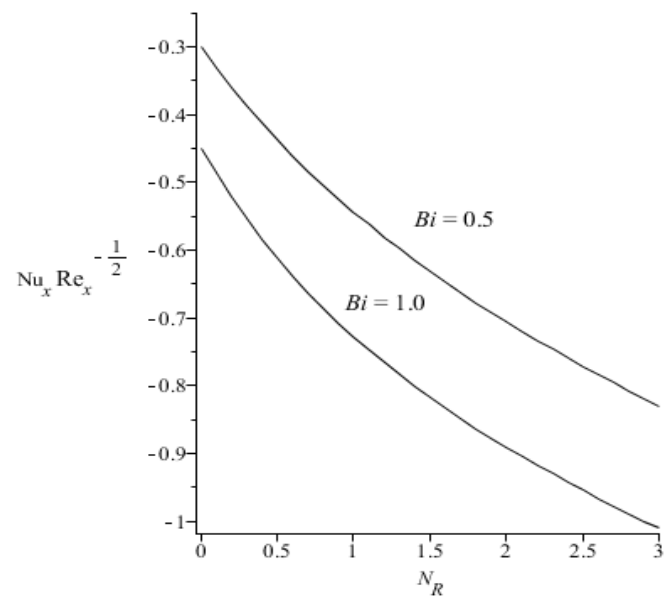

Fig. 13 Variation of the $N u_{x} \mathrm{Re}_{x}^{-1 / 2}$ with $N_{R}$ for various values of $\mathrm{Bi}$.

Table 2 Numerical values of $C_{f} \mathrm{Re}_{x}^{1 / 2}$ for various values of physical parameters.

\begin{tabular}{cccc}
\hline$\lambda$ & $\lambda_{2}$ & $M$ & $C_{f} \mathrm{Re}_{x}^{1 / 2}$ \\
\hline 0.1 & 0.1 & 0.1 & -1.04880885 \\
0.2 & 0.1 & 0.1 & -1.00415803 \\
0.3 & 0.1 & 0.1 & -0.96474638 \\
0.4 & 0.1 & 0.1 & -0.92966968 \\
0.4 & 0.2 & 0.1 & -0.97100831 \\
0.4 & 0.3 & 0.1 & -1.01065750 \\
0.4 & 0.4 & 0.1 & -1.04880885 \\
0.4 & 0.4 & 0.2 & -1.09544512 \\
0.4 & 0.4 & 0.3 & -1.14017543 \\
0.4 & 0.4 & 0.4 & -1.18321596 \\
\hline
\end{tabular}


Table 3 Numerical values of $N u_{x} \mathrm{Re}_{x}^{-1 / 2}$ for various values of physical parameters.

\begin{tabular}{cccccccc}
\hline$B i$ & $\lambda$ & $\lambda_{2}$ & $\operatorname{Pr}$ & $N_{R}$ & $E c$ & $M$ & $N u_{x} \mathrm{Re}_{x}^{-1 / 2}$ \\
\hline 0.5 & 0.1 & 0.1 & 0.71 & 0.2 & 0.2 & 0.1 & -0.38926871 \\
1.0 & 0.1 & 0.1 & 0.71 & 0.2 & 0.2 & 0.1 & -0.57505679 \\
1.4 & 0.1 & 0.1 & 0.71 & 0.2 & 0.2 & 0.1 & -0.66585560 \\
2.0 & 0.1 & 0.1 & 0.71 & 0.2 & 0.2 & 0.1 & -0.75529924 \\
2.0 & 0.2 & 0.1 & 0.71 & 0.2 & 0.2 & 0.1 & -0.74831132 \\
2.0 & 0.4 & 0.1 & 0.71 & 0.2 & 0.2 & 0.1 & -0.73493100 \\
2.0 & 1.0 & 0.1 & 0.71 & 0.2 & 0.2 & 0.1 & -0.69898542 \\
2.0 & 1.0 & 0.2 & 0.71 & 0.2 & 0.2 & 0.1 & -0.70839617 \\
2.0 & 1.0 & 0.4 & 0.71 & 0.2 & 0.2 & 0.1 & -0.72405424 \\
2.0 & 1.0 & 1.0 & 0.71 & 0.2 & 0.2 & 0.1 & -0.75529924 \\
2.0 & 1.0 & 1.0 & 1.0 & 0.2 & 0.2 & 0.1 & -0.87179816 \\
2.0 & 1.0 & 1.0 & 5.0 & 0.2 & 0.2 & 0.1 & -1.39673468 \\
2.0 & 1.0 & 1.0 & 7.0 & 0.2 & 0.2 & 0.1 & -1.49274065 \\
2.0 & 1.0 & 1.0 & 7.0 & 0.4 & 0.2 & 0.1 & -1.74198047 \\
2.0 & 1.0 & 1.0 & 7.0 & 0.6 & 0.2 & 0.1 & -1.97857064 \\
2.0 & 1.0 & 1.0 & 7.0 & 0.8 & 0.2 & 0.1 & -2.20421759 \\
2.0 & 1.0 & 1.0 & 7.0 & 0.8 & 0.3 & 0.1 & -2.12467015 \\
2.0 & 1.0 & 1.0 & 7.0 & 0.8 & 0.4 & 0.1 & -2.04512263 \\
2.0 & 1.0 & 1.0 & 7.0 & 0.8 & 0.5 & 0.1 & -1.96557474 \\
2.0 & 1.0 & 1.0 & 7.0 & 0.8 & 0.5 & 0.2 & -1.93192362 \\
2.0 & 1.0 & 1.0 & 7.0 & 0.8 & 0.5 & 0.3 & -1.89901460 \\
2.0 & 1.0 & 1.0 & 7.0 & 0.8 & 0.5 & 0.4 & -1.86678146 \\
\hline
\end{tabular}

\section{CONCLUSION}

The present study has shown how the influence of radiation parameter and viscous dissipation affected the boundary layer flow of MHD Jeffrey fluid over a stretching sheet. The significant results of this study can be recapitulated as below:

- The ratio of relaxation to retardation times $\lambda$ is dependent on the Deborah number $\lambda_{2}$.

- The ratio of relaxation to retardation times $\lambda$ pronounces the opposite effect to the Deborah number $\lambda_{2}$ for both velocity and temperature profiles.

- The magnetic parameter $M$ exhibit the opposite effect between velocity and temperature profiles and skin friction coefficient and Nusselt number.

- The velocity profile for $E c$ and $N_{R}$ is unique.

- The skin friction coefficient for radiation $N_{R}$ and biot number $B i$ is also unique.

\section{ACKNOWLEDGEMENT}

This work was financially supported by the Universiti Malaysia Pahang (UMP) under the Research University Grant (PGRS1703100, RDU170358 and RDU141306).

\section{REFERENCES}

Afridi, M. I., Qasim, M., Khan, I., Shafie, S., and Alshomrani, A. S. (2016). Entropy generation in magnetohydrodynamic mixed convection flow over an inclined stretching sheet. Entropy, 19(1), 10.

Al-Sharif, H. A. M., Kasim, A. R. M., Salleh, M. Z., Mohammad, N. F., Shafie, S., and Ali, A. (2016). Influence of slip velocity on convective boundary layer flow of Jeffrey fluid under convective boundary conditions. ARPN Journal of Engineering and Applied Sciences, 11(18), 10950-10953.

Babu, D. H., and Narayana, P. V. S. (2016). Joule heating effects on MHD mixed convection of a Jeffrey fluid over a stretching sheet with power law heat flux: A numerical study. Journal of Magnetism and Magnetic Materials, 412(2016), 185-193.

Bataller, R. C. (2008). Radiation effects in the Blasius flow. Applied Mathematics and Computation, 198(1), 333-338.
Crane, L. J. (1970). Flow past a stretching plate. Zeitschrift für angewandte Mathematik und Physik ZAMP, 21(4), 645-647.

Dalir, N. (2014). Numerical study of entropy generation for forced convection flow and heat transfer of a Jeffrey fluid over a stretching sheet. Alexandria Engineering Journal, 53(4), 769-778.

Das, K., Acharya, N., and Kundu, P. K. (2015). Radiative flow of MHD Jeffrey fluid past a stretching sheet with surface slip and melting heat transfer. Alexandria Engineering Journal, 54(4), 815-821.

Erickson, L., Fan, L., and Fox, V. (1966). Heat and mass transfer on moving continuous flat plate with suction or injection. Industrial \& Engineering Chemistry Fundamentals, 5(1), 19-25.

Farooq, M., Gull, N., Alsaedi, A., and Hayat, T. (2015). MHD flow of a Jeffrey fluid with Newtonian heating. Journal of Mechanics, 31(03), 319-329.

Gupta, P., and Gupta, A. (1977). Heat and mass transfer on a stretching sheet with suction or blowing. The Canadian Journal of Chemical Engineering, 55(6), 744-746.

Hayat, T., Asad, S., Mustafa, M., and Alsaedi, A. (2015). MHD stagnation-point flow of Jeffrey fluid over a convectively heated stretching sheet. Computers \& Fluids, 108, 179-185.

Hayat, T., Iqbal, Z., Mustafa, M., and Alsaedi, A. (2014). Unsteady flow and heat transfer of Jeffrey fluid over a stretching sheet. Therm. Sci, 18(4), 1069-1078.

Magyari, E., and Keller, B. (1999). Heat and mass transfer in the boundary layers on an exponentially stretching continuous surface. Journal of Physics D: Applied Physics, 32(5), 577.

Merkin, J. (1994). Natural-convection boundary-layer flow on a vertical surface with Newtonian heating. International Journal of Heat and Fluid Flow, 15(5), 392-398.

Mohamed, M. K. A., Noar, N. A. Z. M., Salleh, M. Z., and Ishak, A. (2016). Free convection boundary layer flow on a horizontal circular cylinder in a nanofluid with viscous dissipation. Sains Malaysiana, 45(2), 289-296.

Mohamed, M. K. A., Salleh, M. Z., Ishak, A., and Pop, I. (2015). Stagnation point flow and heat transfer over a stretching/shrinking sheet in a viscoelastic fluid with convective boundary condition and partial slip velocity. The European Physical Journal Plus, 130(8), 171.

Molla, M. M., Hossain, M. A., and Paul, M. C. (2006). Natural convection flow from an isothermal horizontal circular cylinder in presence of heat generation. International Journal of Engineering Science, 44(13-14), 949958 .

Mustafa, M., Hayat, T., and Hendi, A. A. (2012). Influence of Melting Heat Transfer in the Stagnation-Point Flow of a Jeffrey Fluid in the Presence of Viscous Dissipation. Journal of Applied Mechanics, 79(2), 024501024505 .

Narayana, P. V. S., and Babu, D. H. (2016). Numerical study of MHD heat and mass transfer of a Jeffrey fluid over a stretching sheet with chemical reaction and thermal radiation. Journal of the Taiwan Institute of Chemical Engineers, 59(2016), 18-25.

Qasim, M. (2013). Heat and mass transfer in a Jeffrey fluid over a stretching sheet with heat source/sink. Alexandria Engineering Journal, 52(4), 571575 .

Sakiadis, B. (1961). Boundary- layer behavior on continuous solid surfaces: I. Boundary- layer equations for two- dimensional and axisymmetric flow. AIChE Journal, 7(1), 26-28.

Salleh, M. Z., Nazar, R., and Pop, I. (2010). Boundary layer flow and heat transfer over a stretching sheet with Newtonian heating. Journal of the Taiwan Institute of Chemical Engineers, 41(6), 651-655.

Zokri, S. M., Arifin, N. S., Mohamed, M. K. A., Salleh, M. Z., Kasim, A. R. M., and Mohammad, N. F. (2016). Numerical solutions on mixed convection boundary layer and heat transfer of Jeffrey fluid over a horizontal circular cylinder by using Keller-box method. The National Conference for Postgraduate Research 2016. Universiti Malaysia Pahang, 913-926. 